Results Detailed images of the basilar artery, lower cerebellar arteries, basilar perforators, vertebrobasilar junction, spinal perforators were obtained (figure 1). Due to the high resolution of the method, the layers of the vessel wall were imaged. What was even more interesting was the ability to image beyond the artery into the structural components of the subarachnoid space with the arachnoid membranes and what we believe to be the arachnoid trabeculae. Venous structures and nerve root origins were also recognized.

Conclusion HF-OCT imaging can offer detailed visualization not only of the vessel wall, the branching pattern of basilar and spinal perforators but also of structures of the subarachnoid space impossible to study with other imaging modalities due to inadequate resolution. The clinical implication of such an analysis is yet to be discovered.

Disclosures V. Anagnostakou: None. Z. Vardar: None. A. Puri: None. G. Ughi: None. M. Gounis: None.

\section{E-016 A MODIFIED RABBIT ELASTASE INDUCED ANEURYSM THAT SHOWS CONTINUED INFLAMMATION AND GROWTH}

${ }^{1,2} \mathrm{R}$ King*, ${ }^{1} \mathrm{~J}$ Caroff, ' $\mathrm{A}$ Leopardi, ${ }^{1} \mathrm{~A}$ Bogdanov, ${ }^{1} \mathrm{M}$ Gounis. ${ }^{1}$ Radiology, University of Massachusetts Medical School, Worcester, MA; ${ }^{2}$ Biomedical Engineering, Worcester Polytechnic Institute, Worcester, MA

\subsection{6/neurintsurg-2020-SNIS.52}

Introduction Animal models of saccular aneurysms are stable following creation, and do not actively remodel or rupture. Recently, rodent models of aneurysm wall remodeling and rupture have been described by surgical grafting of decellularized arterial tissue. ${ }^{1}$ We sought to 1) develop and apply a modified in situ decellularization approach, not require complex grafting procedures, in a large animal model of saccular aneurysms and 2) investigate modified aneurysmal expansion and inflammation using in vivo imaging.

Methods 18 New Zealand White rabbits (3.0-3.5 kg) underwent standard elastinolysis aneurysm creation of the right common carotid artery. The animals were randomized

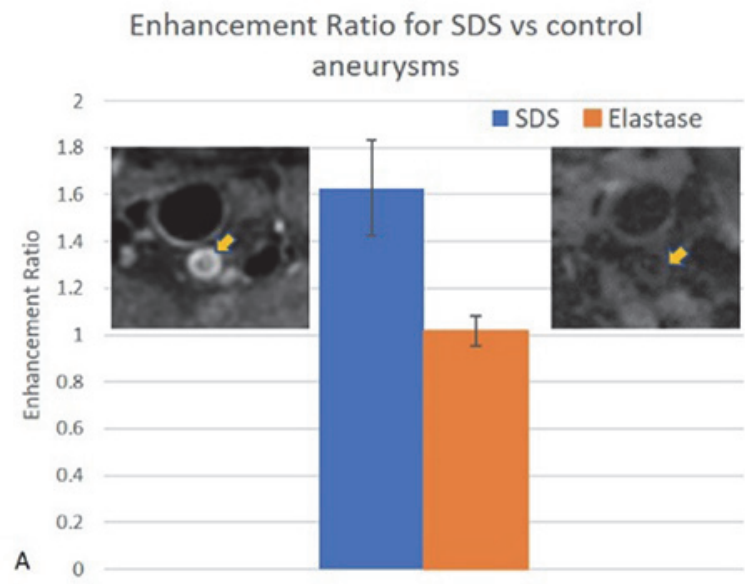

2:1 to receive isolated vessel SDS treatment (perfusion with $1 \%$ solution for $45 \mathrm{~min}$ ) or nothing. Every two weeks until week 8 , three of the rabbits (2 SDS+elastase, 1 elastase), were subjected to MR imaging, ${ }^{2}$ followed by contrast injection with stable macrocyclic gadolinium(III)-based contrast agent that has been shown to selectively enhance the areas of vessel wall myeloperoxidase (MPO) accumulation. ${ }^{3}$ 4 The MRI was repeated at 3 hours post contrast injection and the enhancement ratio (ER) was calculated. All aneurysms were measured for size by digital subtraction angiography.

Results Of the 17 rabbits included in the study, 11 were treated in situ with SDS following elastase incubation and 6 served as controls. One SDS-treated rabbit was excluded due to rupture of the carotid artery during recovery. During follow-up MR imaging the ER was greater than 1 for all SDStreated animals, whereas the controls had an ER of greater than 1 only at the two-week timepoint (consistent with the expected healing process). Average ER for SDS-treated animals was $1.63 \pm 0.20$, while control animals had an ER of 1.02 $\pm 0.06(\mathrm{p}<0.001)$. SDS-treated aneurysms width increased by over $45 \%$ over the first 8 weeks, while the control aneurysms grew about $20 \%$ in the first 2 weeks and then remained stable.

Conclusions During the 12 weeks post aneurysm creation, MPO-mediated MR signal enhancement was continuously detected within the wall of the aneurysm, and other than at 2 weeks, only in the SDS-treated animals. This novel modified large animal aneurysm model is expected to be useful for testing of new therapies that rely on not only the mechanical properties of the aneurysm but also the biological processes that make aneurysms vulnerable to rupture.

\section{REFERENCES}

1. Stroke 2014;45:248-254.
2. AJNR 2015;36:146-52.
3. Radiology 2009;252:696-703.
4. Sci Rep 2018;8:7687.

Disclosures R. King: None. J. Caroff: 1; C; FullBright. A. Leopardi: None. A. Bogdanov: None. M. Gounis: None.

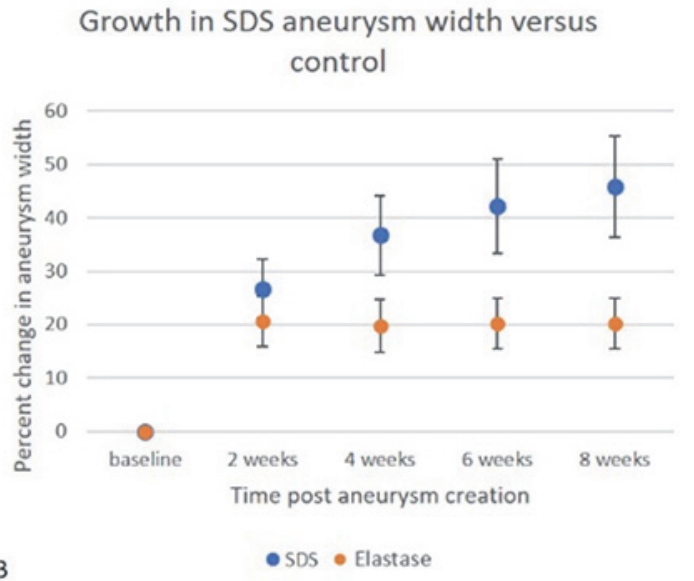

Abstract E-016 Figure 1 A) overall enhancement ration of SDS vs control aneurysms, showing a significant difference $(p<0.001)$, The left subpanel shows an SDS treated aneurysm (arrow) after contrast injection, right subpanel shows a control aneurysm (arrow) after contrast injection. B) Growth in the width of the aneurysm width over time. After two weeks, the control aneurysm stabilize in size, while the SDS aneurysms continue to grow. Due to reduction in numbers at each time point, standard deviation increases at each follow up timepoint 